\title{
A model of mid-latitude E-region plasma convergence inside a planetary wave cyclonic vortex
}

\author{
S. Shalimov ${ }^{1}$ and C. Haldoupis \\ Physics Department, University of Crete, Iraklion, Crete, 710 03, Greece \\ ${ }^{1}$ permanently at the Institute of Physics of the Earth, Moscow, Russia
}

Received: 12 December 2001 - Revised: 2 March 2002 - Accepted: 12 March 2002

\begin{abstract}
Recently, Shalimov et al. (1999) proposed a new mechanism for large-scale accumulation of long-lived metallic ions in the mid-latitude ionosphere driven by planetary waves in the lower thermosphere. In this mechanism, the combined action of frictional and horizontal magnetic field forces at E-region altitudes causes the plasma to converge and accumulate in large areas of positive neutral wind vorticity within a propagating planetary wave. The present paper provides a theoretical formulation for this mechanism by modelling both horizontal and vertical plasma transport effects within a planetary wave vortex, of cyclonic neutral wind. Non-steady-state numerical solutions of the ion continuity equation show that the proposed accumulation process can enhance the ionization significantly inside the planetary wave vortex but its efficiency depends strongly on altitude, whereas on the other hand, it can be complicated by vertical plasma motions. The latter, which are driven by the same planetary wave wind field under the action of the vertical Lorentz force and meridional wind forcing along the magnetic field lines, can lead to either plasma compressions or depletions, depending on the prevailing wind direction. We conclude that, for shorter times, vertical plasma transport may act constructively to the horizontal gathering process to produce considerable E-region plasma accumulation over large sectors of a planetary wave vortex of cyclonic winds.
\end{abstract}

Key words. Ionosphere (ionosphere-atmosphere interactions; mid-latitude ionosphere; sporadic E-layers) - Meteorology and atmospheric dynamics (waves and tides)

\section{Introduction}

Recent observations suggested that planetary waves could modulate the occurrence of mid-latitude E-region coherent backscatter observed with HF and VHF radars during conditions of strong sporadic E-layers $\left(E_{S}\right)$ in the summer night-

Correspondence to: C. Haldoupis (chald@ physics.uoc.gr) time (Tsunoda et al., 1998; Voiculescu et al., 1999, 2000). More recently, Haldoupis and Pancheva (2002) provided, for first time, convincing experimental evidence showing that planetary waves play a direct role in the physics of sporadic E-layers. In earlier studies, long-term periodicities in electron concentration measured with various techniques in the mid-latitude ionosphere were also attributed to planetary waves (e.g. Lastovicka et al., 1994; Pancheva et al., 1994; Apostolov et al., 1995; Fraser, 1977; Zhou, 1998, and more references cited therein). Planetary waves (PW) are global scale quasi-periodic oscillations in temperature, density, pressure and wind, with periods near 2, 5, 10 and 16 days, which prevail in the mesosphere and lower thermosphere (MLT), i.e. in altitudes from about 80 to $150 \mathrm{~km}$ (e.g. see Forbes, 1994).

Prompted by the recent findings, which indicated that PW can play a role in the generation of mid-latitude sporadic Elayers, Shalimov et al. (1999) (hereafter called "paper A") proposed a new mechanism for large-scale plasma accumulation in the mid-latitude E-region ionosphere driven by $\mathrm{PW}$ kinetic energy. In this process, long-lived metallic ions are forced to converge and accumulate inside large areas of horizontal wind shears within a cyclonic vortex of a propagating PW. In its simplest form, this model resembles the wellknown east-west vertical wind shear mechanism of $E_{s}$ formation (Whitehead, 1961; Axford, 1963), but with the geometry now applied in the horizontal plane.

In introducing the model, paper A placed the emphasis on the physical base of the proposed mechanism and provided a simplified theory which considered only steady-state conditions in the horizontal north-south direction. The present paper, which is a continuation of this first work, provides a more rigorous mathematical formulation of the PW accumulation process and presents non-steady-state numerical results of ion convergence in the horizontal plane, as well as estimates of the vertical plasma transport effects. 


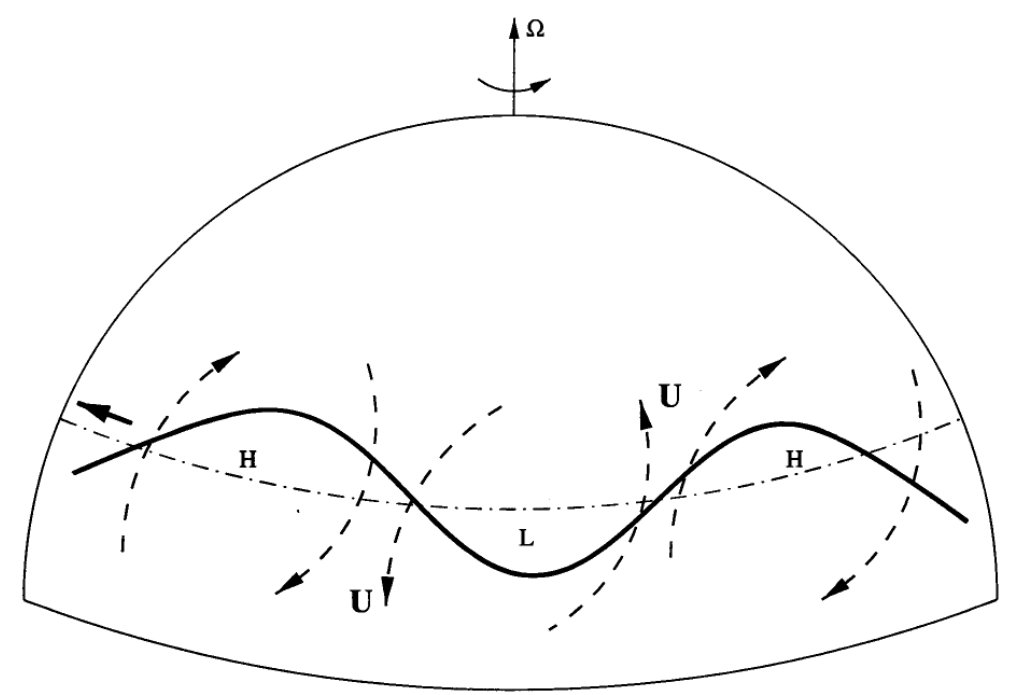

Fig. 1. Visualization of a planetary wave in the Northern Hemisphere (from Shalimov et al., 1999, based on Holton, 1982).

\section{The PW plasma accumulation process}

Before treating the problem theoretically, we provide here a brief physical description of the mechanism. The basic idea relies on the concept of a PW, as shown in Fig. 1, which is based on the PW visualization provided in the textbook by Holton (1982). In a barotropic atmosphere under geostrophic balance, a westward propagating PW is accompanied by large cyclonic and anticyclonic vortices that develop around regions of low and high pressure, which set up large-scale alternating horizontal wind shears in successive regions of positive and negative vorticity.

Since PW are present on a regular basis in the MLT region, the neutral wind vortices in Fig. 1 can have an effect on redistributing long-lived metallic ion plasma in the Eregion. This is due to the fact that ion motion is controlled by both the ion-neutral collisional force and the magnetic field (Lorentz) force, with this force ratio depending on $v_{i} / \Omega_{i}$, i.e. the ion-neutral collision frequency to ion gyrofrequency ratio. At E-region altitudes, the ions drift in the horizontal plane along with the neutral wind $\boldsymbol{U}$, but they are also gradually deflected under the action of the horizontal Lorentz force $\boldsymbol{U} \times \boldsymbol{B}_{z}$ (where $\boldsymbol{B}_{z}$ is the vertical magnetic field component), so they converge inward inside PW cyclonic vortices and diverge outward from areas occupied by anticyclonic motions. Consequently, metallic ion plasma density will tend to increase inside cyclonic vortices and decrease inside anticyclonic ones. This idealized picture, however, is in reality more complex due to the vertical ion transport caused by the horizontal magnetic field Lorentz forces and neutral wind components parallel to the magnetic field. These effects, which were mentioned but not treated in paper A, will now be taken into consideration in the present work.

Finally, we stress that the Shalimov et al. (1999) PW mechanism of horizontal plasma accumulation does not lead by itself to direct sporadic $E$ layer formation. The latter re- quires, in line with $E_{s}$ theory (Whitehead, 1961; Axford, 1963), the presence of vertical wind shears which are believed to be provided by shorter scale wind systems, such as gravity waves and/or tidal motions (e.g. see review by Whitehead, 1989). The PW mechanism acts indirectly in $E_{s}$ formation, i.e. the narrow layers are expected to form (under the action of a short-scale vertical wind shear) more easily and be stronger inside planetary wave areas of positive (cyclonic) vorticity, where metallic ion density is higher, rather than in areas of negative (anticyclonic) vorticity, where metallic ion concentration is lower.

\section{Theory}

In paper $\mathrm{A}$, the $\mathrm{PW}$ accumulation process was treated in analogy with the one-dimensional vertical wind shear theory of mid-latitude $E_{s}$ formation proposed by Whitehead (1961). Paper A considered only horizontal Lorentz forces, driven by the vertical magnetic field component $B_{z}$, to act upon the meridional component of a PW cyclonic wind shear; this approach led to a simplified expression for steady-state conditions which provided only a general insight into the problem. In the following, a more detailed theoretical treatment of the mechanism is presented which considers a two-dimensional, vortex-like, geometry and arrives at non-steady-state numerical estimates for the temporal and spatial scales of the plasma gathering process. Furthermore, the re-distribution of ionization in altitude is also studied by taking into consideration both the vertical Lorentz force and neutral wind ion forcing parallel to the magnetic field. Since PW have very large horizontal scales (several thousands of $\mathrm{km}$ ) relative to their vertical extent ( $\mathrm{a}$ few tens of $\mathrm{km}$ ), in the following, the horizontal and vertical ion motions are treated separately due to their grossly different scales in space and time. 


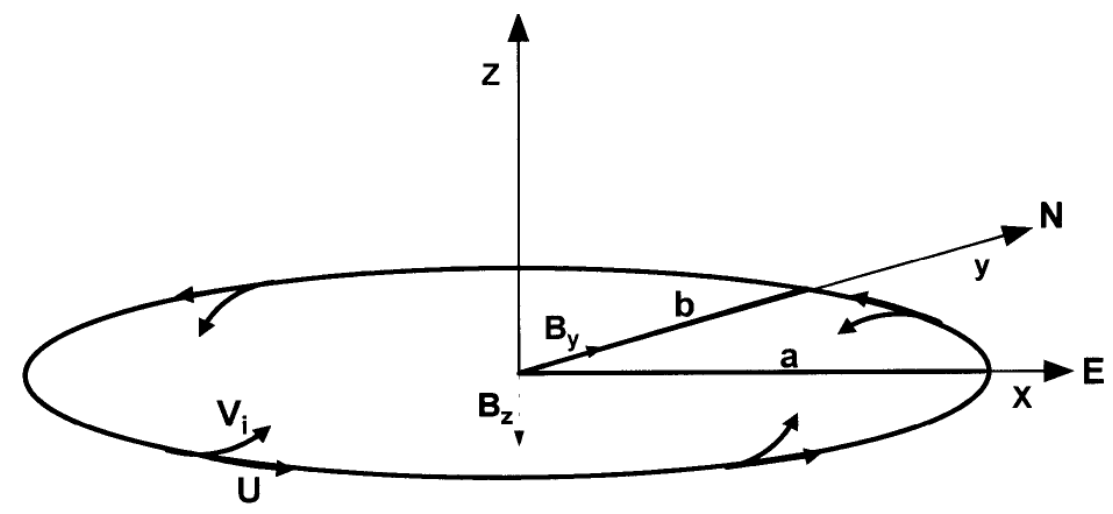

Fig. 2. An ellipse with large eccentricity used in the present model as representative of a planetary wave vortex of cyclonic winds. In E-region, the ions move with the wind but also are subject to $\boldsymbol{U} \times \boldsymbol{B}$ Lorentz forces. Also shown here is the local orthogonal coordinate system used in the calculations.

\subsection{Horizontal accumulation}

Since zonal scale lengths in PW are larger than meridional ones, the vortices in Fig. 1 are best represented by ellipses with large eccentricities $e^{2}=1-(a / b)^{2}$. In the analysis, we adopt a horizontal elliptical cyclonic vortex centered at midlatitudes, as shown in Fig. 2 in a local Cartesian coordinate system with its $x$ and $y$ axes pointing to the east and north, respectively. At E-region altitudes, neutrals move with the horizontal velocity $\boldsymbol{U}$ along the ellipse $(y / a)^{2}+(x / b)^{2}=1$ in the plane $z=$ const, i.e. we take $U_{z}=0$ in the altitude range of interest from, say, 100 to $125 \mathrm{~km}$.

For the ion velocity, $\boldsymbol{V}_{i}$, we use the well-known general relation which follows from the ion momentum balance equation (e.g. see Gershman, 1974; Kelley, 1989)

$$
\begin{aligned}
\boldsymbol{V}_{i}= & \frac{1}{1+\beta_{i}^{2}}\left[\boldsymbol{U}+\beta_{i}(\boldsymbol{U} \times \boldsymbol{b})+\beta_{i}^{2} \boldsymbol{b}(\boldsymbol{U} \cdot \boldsymbol{b})\right. \\
& -\frac{T_{i}}{m_{i} \Omega_{i}} \beta_{i}\left(\frac{\nabla N}{N}+\beta_{i}\left(\frac{\nabla N}{N} \times \boldsymbol{b}\right)+\beta_{i}^{2} \boldsymbol{b}\left(\frac{\nabla N}{N} \cdot \boldsymbol{b}\right)\right) \\
& \left.+\frac{e}{m_{i} \Omega_{i}} \beta_{i}\left(\boldsymbol{E}+\beta_{i}(\boldsymbol{E} \times \boldsymbol{b})+\beta_{i}^{2} \boldsymbol{b}(\boldsymbol{E} \cdot \boldsymbol{b})\right)\right]
\end{aligned}
$$

where $\beta_{i}=\Omega_{i} / \nu_{i}, \boldsymbol{b}=\boldsymbol{B}_{0} /\left|\boldsymbol{B}_{0}\right|$ is the Earth's magnetic field unit vector, $\boldsymbol{U}$ is the neutral wind velocity, $\boldsymbol{E}$ is the total (ambient plus polarization) electric field, $N$ is the plasma density, $v_{i}$ and $\Omega_{i}$ are the ion-neutral collision frequency and gyrofrequency, respectively, $m_{i}$ is the ion mass, and $T_{i}$ is the ion temperature.

Next, the Cartesian coordinates are transformed to axisymmetrical ones by using transformations $X=(a / b) x, Y=y$, $Z=z$, and taking cylindrical coordinates $(r, \phi, z)$, with the $z$ axis directed vertically upward along the axis of symmetry. By assuming there is only $r$-dependence, and considering only long-lived metallic ions (i.e. we ignore the ionization and recombination terms), we substitute the radial component of the ion velocity from Eq. (1) into the continuity equation to obtain:

$$
\begin{array}{r}
\frac{\partial N}{\partial t}+\frac{1}{r} \frac{\partial}{\partial r}\left\{\frac { r } { 1 + \beta _ { i } ^ { 2 } } \left[\beta_{i} b_{z} N U_{\phi}+\beta_{i}^{2} b_{r} b_{\phi} U_{\phi}-\frac{T_{i} \beta_{i}}{m_{i} \Omega_{i}}\right.\right. \\
\left.\left.\left(1+\beta_{i}^{2} b_{r}^{2}\right) \frac{\partial N}{\partial r}+\frac{e \beta_{i}}{m_{i} \Omega_{i}}\left(1+\beta_{i}^{2} b_{r}^{2}\right) N E_{r}\right]\right\}=0
\end{array}
$$

where $b_{r}, b_{\phi}, b_{z}$, are the components of the magnetic field unit vector in the cylindrical coordinate system. Note that for simplicity we take $U_{r}=0$.

Next, we ignore the ambient electric field but consider polarization fields by taking instead of the ion diffusion term $D_{i}=K_{B} T_{i} / m_{i} v_{i}$ the ambipolar diffusion coefficient $D_{a}=$ $K_{B}\left(T_{e}+T_{i}\right) / m_{i} v_{i}$, where $T_{e}$ is the electron temperature and $K_{B}$ is the Boltzmann constant. Note that for E-region altitudes between 100 and $125 \mathrm{~km}$, we take $\beta_{e}^{2} \gg \beta_{i}^{2}$ and $\beta_{e}^{2} b_{r}^{2} \gg 1$, where $\beta_{e}=\Omega_{e} / v_{e}$ is the ratio of electron gyrofrequency to the electron-neutral collision frequency. Also, in this altitude range, we ignore terms with $\beta_{i}^{2}$ as rather smaller than 1.0 (e.g. at $120 \mathrm{~km} \beta_{i} \simeq 0.57$ and for a typical midlatitude dip angle $I=50^{\circ}$, then $\beta_{i}^{2} \sim 0.3$ and $\beta_{i}^{2} b_{r}^{2} \leq 0.13$ ). Therefore, we believe that, by omitting all the terms which are proportional to $\beta_{i}^{2}$ in the above equation, we provide here a reasonable approximation for a purely horizontal and axisymmetrical plasma convergence process, i.e. radially towards the center of the PW wind vortex, under the action of the vertical component of the magnetic field only, which is also taken as approximately constant inside the PW vortex.

Therefore, the continuity equation is simplified as follows

$\frac{\partial N}{\partial t}+\beta_{i} b_{z} \frac{1}{r} \frac{\partial}{\partial r}\left(r N U_{\phi}\right)=D_{a} \frac{1}{r} \frac{\partial}{\partial r}\left(r \frac{\partial N}{\partial r}\right)$.

Next, we adopt a sinusoidal form $U_{\phi}=U_{0} \sin \left(k_{v} r\right)$ for the PW cyclonic vortex, where $k_{v}=2 \pi / \lambda_{v}$ is an effective "wave number" of the cyclonic wind pattern. Also, we define new dimensionless variables, i.e. $\tau=t \cdot\left(k_{v} U_{0} \beta_{i} \sin I\right)=$ $t / \tau_{W}$, where $\tau_{W}=1 /\left(k_{v} U_{0} \beta_{i} \sin I\right)$ is the characteristic time of the wind shear plasma converging process, $\delta=$ 


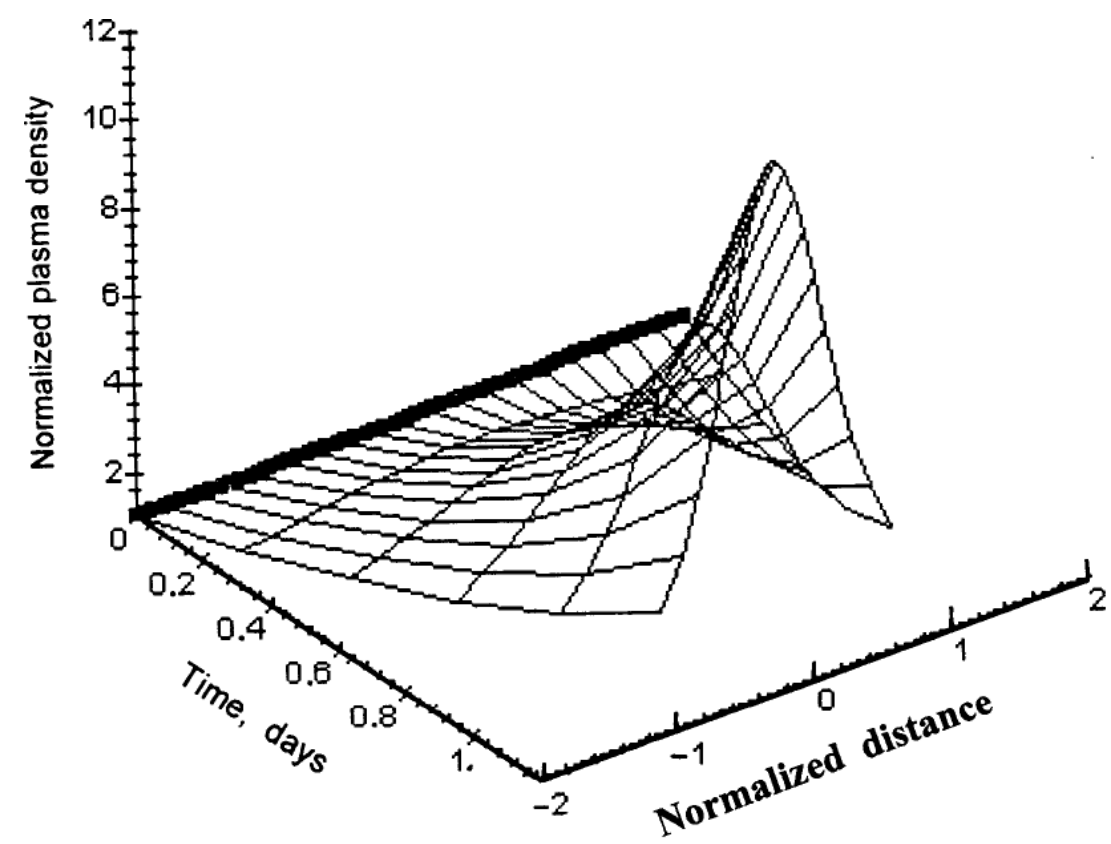

Fig. 3. Non-steady-state numerical solutions showing horizontal, metallic ion, plasma accumulation across a PW cyclonic vortex. It refers to $120 \mathrm{~km}$ and applies for a quasi 2-day PW having a typical cyclonic scale size $\lambda_{v} \sim 5 \times 10^{3} \mathrm{~km}$ and a wave amplitude $U=25 \mathrm{~m} / \mathrm{s}$. The $x$-axis label "normalized distance" refers to the radial distance from the vortex center divided by $\lambda v$. As seen, after about 1 day, the plasma density in the central vortex areas increases up to about an order of magnitude above the background electron density.

$U_{0} \beta_{i} \sin I / k_{v} D_{a}=\tau_{D} / \tau_{W}$, where $\tau_{D}=1 /\left(k_{v}^{2} D_{a}\right)$ is the characteristic diffusion time which opposes plasma convergence, $n=N / N_{0}$, where $N_{0}$ is the background (metallic ion) plasma density, and $\xi=k_{v} \cdot r$. In this way, the last equation takes the dimensionless form

$\frac{\partial n}{\partial \tau}-\frac{1}{\xi} \frac{\partial}{\partial \xi}(n \xi \sin \xi)=\frac{1}{\xi} \frac{\partial}{\partial \xi}\left(\frac{1}{\delta} \xi \frac{\partial n}{\partial \xi}\right)$.

Steady-state solution. A steady-state solution of Eq. (4) can be obtained for $\tau \gg 1$, or $t \gg \tau_{W}$, by applying the same procedure as in the wind shear theory of Axford (1963). This gives

$n(\xi)=n_{0} \exp (\delta \cos \xi)$.

The constant of integration $n_{0}$ can be determined from conservation of total charge particle content in the region occupied by the planetary wave, so that finally

$n(\xi)=I_{0}(\delta) \exp (\delta \cos \xi) \approx \sqrt{2 \pi \delta} \exp [\delta(\cos \xi-1)]$,

where an asymptotic representation for the Bessel function $I_{0}(\delta)$ was used in the limit of $\delta \gg 1$. The last equation can be used to estimate the maximum plasma density reached by the plasma accumulation process under consideration; this is $n_{\max }=\sqrt{2 \pi \delta}$ and it will be attained when $\delta \gg 1$.

Using dimensional variables we can rewrite the steadystate solution of Eq. (4) as

$$
\begin{aligned}
& N(r)=N_{0} \sqrt{2 \pi \delta} \exp \\
& \quad\left\{\delta\left[\cos \left(\frac{2 \pi}{\lambda} \sqrt{\left(\frac{a}{b} x\right)^{2}+y^{2}}\right)-1\right]\right\} .
\end{aligned}
$$

For the case that the eccentricity is near 1, i.e. for an elliptical vortex strongly elongated in the east-west direction (when $b \gg a$ in Fig. 2), Eq. (7) reduces to

$$
N(y)=N_{0} \sqrt{2 \pi \delta} \exp \left[\delta\left(\cos \left(\frac{2 \pi}{\lambda} y\right)-1\right)\right] .
$$

This expression is the same as the one obtained in paper A by using only a meridional PW wind shear. A detailed discussion of the steady-state solution is given in paper A.

Non-steady-state solution. We may obtain more realistic estimates of the effective spatial scales of the accumulating process from non-steady-state solutions of the equation

$\frac{\partial n}{\partial \tau}-\frac{1}{\xi} \frac{\partial}{\partial \xi}(n \xi \sin \xi)=0$,

obtained from Eq. (4) when $\tau \leq 1, \delta \gg 1$ or $t \leq \tau_{W} \ll \tau_{D}$, which allow for the right-hand side in Eq. (4) to be omitted. This is justified because the diffusion time $\tau_{D}$ is very large relative to the plasma gathering time $\tau_{W}$ due to the large horizontal scales involved in the accumulation process (for more details, see paper A). Note that in Eq. (9), time is normalized to the characteristic time of plasma accumulation $\tau_{W}=1 /\left(k_{v} U_{0} \beta_{i} \sin I\right)$, which increases with decreasing altitude because $v_{i}$ increases.

The last partial differential equation can be solved numerically by means of using standard software. To parameterize the altitude variations, we assume an exponential law for ion-neutral collisions, i.e. $v_{i}(z)=v_{i 0} \exp (-z / H)$, where the scale height $H \simeq 10 \mathrm{~km}$ and $v_{i 0}=1.9 \times 10^{3} \mathrm{~s}^{-1}$ at $100 \mathrm{~km}$ 


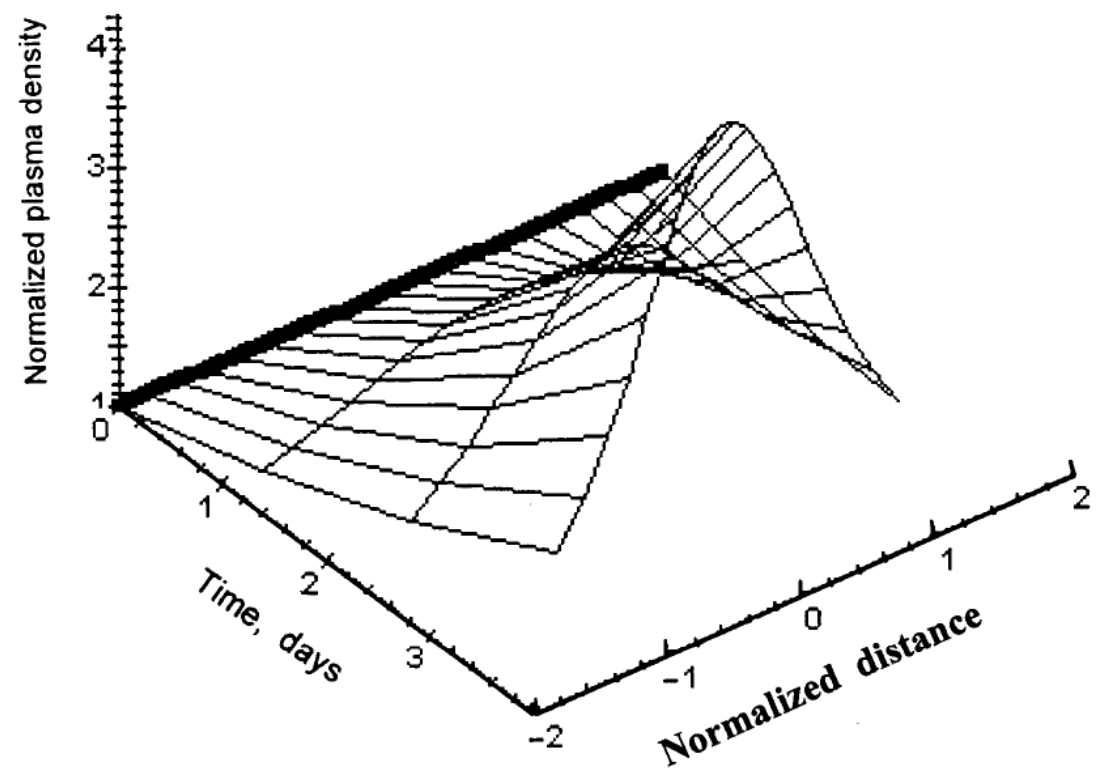

Fig. 4. Same as in Fig. 3 but for a quasi 5-day PW having a typical cyclonic scale size $\lambda_{v} \sim 15 \times 10^{3} \mathrm{~km}$ and a wave amplitude $U=15 \mathrm{~m} / \mathrm{s}$. As seen, after about 2.5 days, the metallic ion plasma density in the central vortex areas increases near 3 times the background density. (e.g. see Kelley, 1989). Also, an ionic mass $m_{i}=42 \mathrm{amu}$ (atomic mass units) is used as a representative of the metallic ion population mean ionic mass (a value between the heavy $\mathrm{Fe}^{+}$ions (56 amu) and lighter metallic ions, such as $\mathrm{Si}^{+}$ (29 amu) and $\mathrm{Mg}^{+}(24 \mathrm{amu})$ ), thus $\Omega_{i}=1.4 \times 10^{2} \mathrm{~s}^{-1}$. Using these parameters and taking, for example, $\lambda_{v}=5 \times 10^{3} \mathrm{~km}$ and $U_{0}=25 \mathrm{~m} / \mathrm{s}$, which we consider as reasonable for a 2-day PW (e.g. see Holton, 1982; Jacobi et al., 1998), it turns out that $\tau_{W}$ ranges from about 6 days at $100 \mathrm{~km}$ to about 1 day at $120 \mathrm{~km}$. As anticipated, the plasma accumulation process is much more efficient at higher than lower E-region altitudes.

Next, numerical results are presented for 2-day and 5-day planetary waves. Here, we use typical cyclonic scale sizes $\lambda_{v}$ of $5 \times 10^{3} \mathrm{~km}$ and $15 \times 10^{3} \mathrm{~km}$, and wave amplitudes of $25 \mathrm{~m} / \mathrm{s}$ and $15 \mathrm{~m} / \mathrm{s}$ (Holton et al., 1982), respectively; also, we take for the non-steady-state calculations an upper limit to which the model was run equal to half the PW period, i.e. about 1 day and 2.5 days for the 2-day and 5-day waves. Figure 3 shows the solution of Eq. (9) that applies for a 2-day wave at $120 \mathrm{~km}$. The time axis is expressed in days, whereas the plasma density and the radial distance about the vortex center are normalized and thus, dimensionless. As seen, the metallic ion plasma accumulates horizontally towards the center of the cyclonic vortex, so that after one day its density increases by a factor of 8 inside a region with $\xi \sim \pm 0.5$, i.e. a radius of about $400 \mathrm{~km}$. Note that, for the same temporal scale at $110 \mathrm{~km}$, the corresponding plasma density increases are about 3 over an area with a radius of $500 \mathrm{~km}$. Figure 4 is similar to Fig. 3, but it now applies for the 5-day wave; as seen, the ion density is enhanced by a factor of 3.5 in about 2.5 days inside a large area centered at the cyclonic vortex and having a radius of about $2.5 \times 10^{3} \mathrm{~km}$. These numerical estimates suggest that the proposed PW accumulation process can operate inside regions of PW positive vorticity, but its efficiency depends on altitude. However, this mechanism alone may not be very efficient at lower E-Region altitudesr, where ion motion is affected strongly by collisions with the neutrals.

Possibly, an additional process exists which drives the plasma from higher to lower altitudes towards the base of the E-region. Several experimental results indicate that such downward plasma motions do take place at mid-latitude (see, for example, Mathews, 1998 and references therein about descending metallic ion layers).

\section{Vertical ion motions}

Given the horizontal wind field $\boldsymbol{U}$ in Fig. 2, ions will also respond to the vertical Lorentz force $\boldsymbol{U} \times \boldsymbol{B}_{y}$, where $B_{y}=B \cos I$ is the northward magnetic field component. This will tend to drive ions vertically downwards (upwards) in the northern (southern) part of the cyclonic vortex, where $\boldsymbol{U}$ has a westward (eastward) component. As for the electrons, these are strongly magnetized and remain unaffected by the neutral wind; therefore, they simply respond to the ion motion by moving along the field lines to neutralize the plasma.

In addition to the Lorentz-driven vertical ion motions, another contribution comes from the meridional neutral wind which forces ions to move along the field lines, as suggested by MacLeod (1966). In this case, a northward (southward) horizontal wind is expected to drive ions downward (upward) in the upper E-region, since at these heights the plasma is constrained to move along field lines. The efficiency of this mechanism becomes minimal in the lower E-region due to a rapid increase in ion-neutral collision frequency.

In the following, the vertical plasma motions are considered in the E-region altitude range from 100 to $140 \mathrm{~km}$, i.e. over a distance much smaller than the horizontal dimensions of the PW vortex. By considering only z-dependence, Eq. (1) 
reduces to

$$
\begin{aligned}
V_{i z} & =\frac{\beta_{i} \cos I}{1+\beta_{i}^{2}}\left(U_{x}-U_{y} \beta_{i} \sin I\right) \\
& +\frac{1+\beta_{i}^{2} \sin ^{2} I}{1+\beta_{i}^{2}}\left(\frac{\beta_{i} e}{m_{i} \Omega_{i}} E_{z}-D_{i} \frac{1}{N} \frac{\partial N}{\partial z}\right),
\end{aligned}
$$

where it was assumed that $U_{z}=0$. Substitution of the last expression into the continuity equation, where again, we ignore ionization and recombination effects and include polarization fields by means of using instead of $D_{i}$ the ambipolar diffusion coefficient $D_{a}=K_{B}\left(T_{e}+T_{i}\right) / m_{i} v_{i}$, it gives

$$
\begin{aligned}
\frac{\partial N}{\partial t} & +\frac{\partial}{\partial z}\left[N \frac{\beta_{i} \cos I}{1+\beta_{i}^{2}}\left(U_{x}-U_{y} \beta_{i} \sin I\right)\right] \\
& =\frac{\partial}{\partial z}\left[\frac{1+\beta_{i}^{2} \sin ^{2} I}{1+\beta_{i}^{2}} \frac{D_{a}}{N} \frac{\partial N}{\partial z}\right] .
\end{aligned}
$$

Next, we express the PW wind components in terms of the angle $\alpha$ which is measured in the horizontal plane counterclockwise from the eastward wind, i.e. we take $U_{x}=$ $U_{0} \cos \alpha$ and $U_{y}=U_{0} \sin \alpha$; for example, $\alpha$ is 0 for eastward, and $\pi / 2$ for northward wind. Note that although $\alpha$ relates to azimuth it should not be identified with the azimuthal angle $\phi$ which, as explained before, is not entering into our model. In the following, $\boldsymbol{U}$ is taken to be independent of altitude and, as in the previous section, $v_{i}$ is taken to decrease exponentially with altitude, which implies that $\beta_{i} \propto \exp (z / H)$, where $H$ is the atmospheric scale height. Thus, the last equation is re-normalized to take the form

$$
\begin{aligned}
\frac{\partial n}{\partial \tau_{v}} & +\frac{\beta_{i}}{1+\beta_{i}^{2}}\left(\cos \alpha-\beta_{i} \sin I \sin \alpha\right) \frac{\partial n}{\partial \zeta} \\
& =-n \frac{\partial}{\partial \zeta}\left[\frac{\beta_{i}}{1+\beta_{i}^{2}}\left(\cos \alpha-\beta_{i} \sin I \sin \alpha\right)\right],
\end{aligned}
$$

where $\tau_{v}$ and $\zeta$ are dimensionless parameters defined as $\left(t U_{0} \cos I\right) / H$ and $z / H$, respectively. Note that the diffusion term in Eq. (11) was omitted because characteristic diffusion times $\tau_{D} \simeq\left(k_{z}^{2} D_{a}\right)^{-1}$ are of the order of 1 day for a 2-day PW (when using a typical vertical wavelength $\lambda_{z} \sim$ $20 \mathrm{~km}$ and $D_{a}=K_{B}\left(T_{e}+T_{i}\right) / m_{i} v_{i}=C_{s}^{2} / v_{i} \simeq 10^{2} \mathrm{~m}^{2} / \mathrm{s}$ if we use a typical ion acoustic speed of $C_{s} \sim 300 \mathrm{~m} / \mathrm{s}$ and $v_{i} \sim 10^{3} s^{-1}$ ). Thus, $\tau_{D}$ is much larger than the characteristic times $\sim H / U_{0} \cos I$ required for vertical plasma transport, which turns out to be less than about $30 \mathrm{~min}$ for $U \sim 10$ to $30 \mathrm{~m} / \mathrm{s}$.

The last equation can be solved numerically to obtain estimates of electron density profiles at a given angle $\alpha$. In this respect, an important question arises about the appropriate time for running a non-steady-state solution. Here, we approximately estimate such a time by assuming it to be shorter than the time required for turbulent mixing in the Eregion, which is of the order of $H^{2}$ divided by the turbulent diffusion coefficient $D_{T}$. By using a typical value for $D_{T} \simeq 10^{4} \mathrm{~m}^{2} / \mathrm{s}$ (e.g. see Gossard and Hooke, 1975) and taking $H \sim 10 \mathrm{~km}$ (e.g. see Kelley, 1989 and atmospheric parameters given therein), one finds a characteristic turbulent

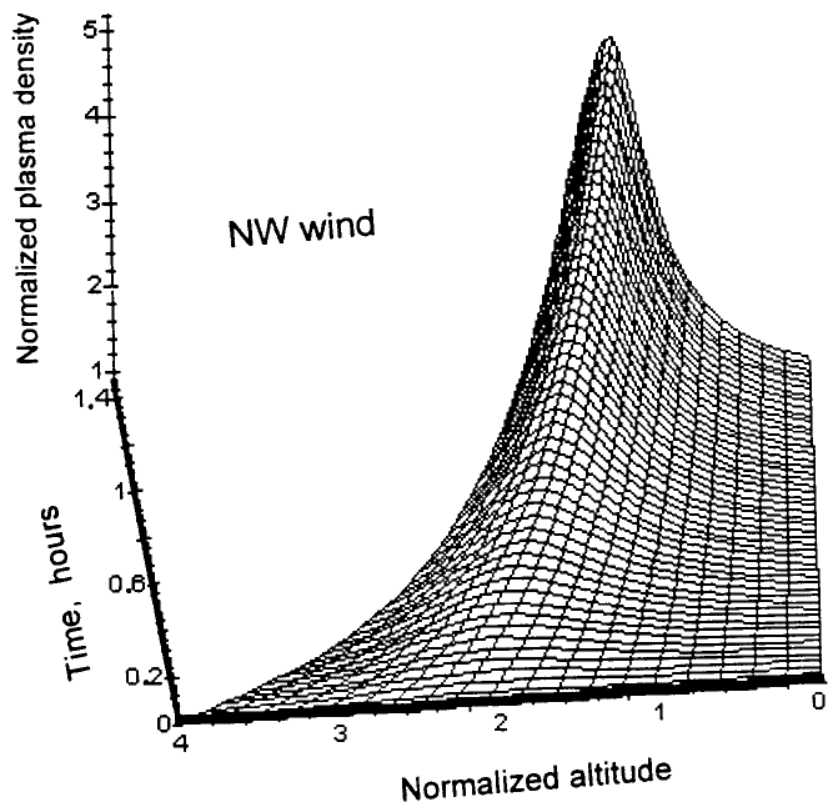

Fig. 5. Typical non-steady-state numerical results of vertical plasma transport inside the north-south PW vortex quadrant (winds point to NE, e.g. see Fig. 2). Ions are driven vertically by the vertical Lorentz force and meridional wind forcing of plasma along the magnetic field lines. These results are based on a quasi 2-day PW wind amplitude of $25 \mathrm{~m} / \mathrm{s}$. The plasma density ( $z$-axis) is normalized to the background mean electron density; the altitude is normalized to a scale height $H=10 \mathrm{~km}$, whereas 0 in the normalized altitude axis corresponds to the bottom height of $100 \mathrm{~km}$. As seen, a broad layer forms in the upper E-region near $130 \mathrm{~km}$. See text for more details.

mixing time of the order of about $2 \mathrm{~h}$. This means that the vertical plasma transport effects are expected to last about two hours and then rebuild again during a time $\tau_{v}$. Following this line of reasoning, we have adopted a characteristic time of one hour for the non-steady-state solution of Eq. (12).

Next, we present and discuss solutions of Eq. (12) for each of the four $90^{\circ}$ sectors in the horizontal plane, e.g. see Fig. 2. Representative non-steady-state solutions are shown for angles of $\alpha=\pi / 4,3 \pi / 4,5 \pi / 4,7 \pi / 4$, which correspond to a wind directed into the northeast (sector I), northwest (sector II), southwest (sector III), and southeast (sector IV), respectively. In the results to be shown, we use $U=25 \mathrm{~m} / \mathrm{s}$. To understand the plasma state in the following figures, one needs to consider the contribution of the collisional and Lorentz terms in the momentum transport equation.

We start with Fig. 5, which applies for sector I, where the wind points to the NE. It shows the distribution of plasma density as a function of time in hours and of normalized altitude distance $z / H$, where $H=10 \mathrm{~km}$ and the bottom height is $100 \mathrm{~km}$. As seen, a plasma layer, having a thickness of $\sim 5 \mathrm{~km}$, forms at about $130 \mathrm{~km}$ after about 1 hour. This results from the combined action of the vertical Lorentz force which pushes ions upward, while at the same time plasma is forced downward along the magnetic field by the northward wind 


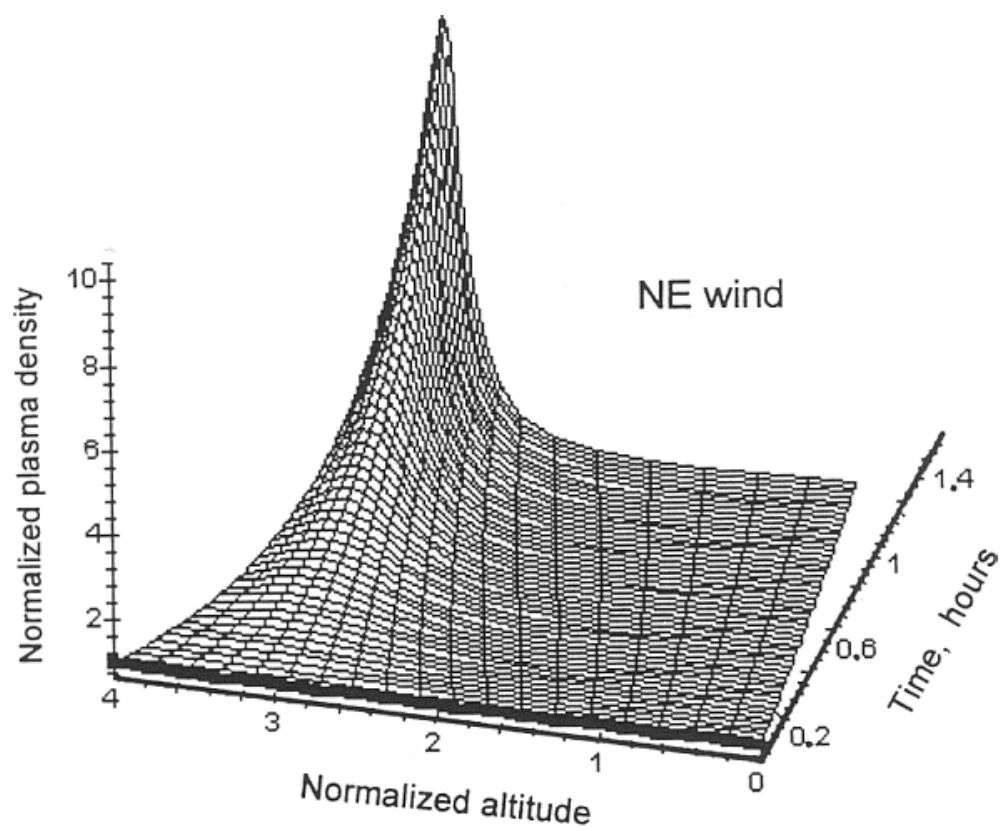

Fig. 6. Same as Fig. 5 but for the northeast PW vortex quadrant, i.e. the profile is representative of PW winds pointing NW. A well-defined broad layer forms near $110 \mathrm{~km}$.

component. These plasma driving forces depend strongly on wind direction. For example, at angles $\alpha \sim 0$ (wind is eastward), the Lorentz force becomes dominant, so that ions are pushed upward in all altitudes; on the other hand, when $\alpha \sim \pi / 2$, the vertical Lorentz force is near zero, since the wind turns nearly northward; therefore, the plasma is constrained to move downward along $\boldsymbol{B}$ untill it is stopped at lower heights by collisions. At angles near $\pi / 4$, as in Fig. 5, plasma motions upward (Lorentz) and downward (northward wind) are comparable; therefore, they combine to form a layer at higher altitudes near $130 \mathrm{~km}$ since their vertical forcing is much more efficient at higher than lower altitudes. It is important to stress that the relative contribution of these two different forces on vertical plasma transport are dependent not only on azimuth but also on altitude.

Figure 6 represents the situation in sector II where the wind points NW. Here, the previous two mechanisms combine to push the ions downward, which results in a considerable plasma accumulation in the lower E-region so that after about an hour, a well-defined plasma layer is formed, peaking near $110 \mathrm{~km}$ and having a thickness of about $\sim 10 \mathrm{~km}$ and a peak density about 5 times the original background density. This is the most favorable quadrant within the cyclonic vortex for vertical plasma accumulation at lower E-region heights, where usually the strongest $E_{s}$ layers are known to be situated (e.g. see Whitehead, 1989; Mathews, 1998).

Figure 7 applies for sector III, where the wind points SW; therefore, its southward component forces the ions upward at higher altitudes, whereas the westward wind Lorentz force drives ions downward to lower altitudes. In this way, the plasma is quickly depleted at higher altitudes, especially due to the efficient southward wind action above 125 to $130 \mathrm{~km}$. As a result, only very little plasma reaches the lower heights, where the effect of both forces diminishes due to enhanced

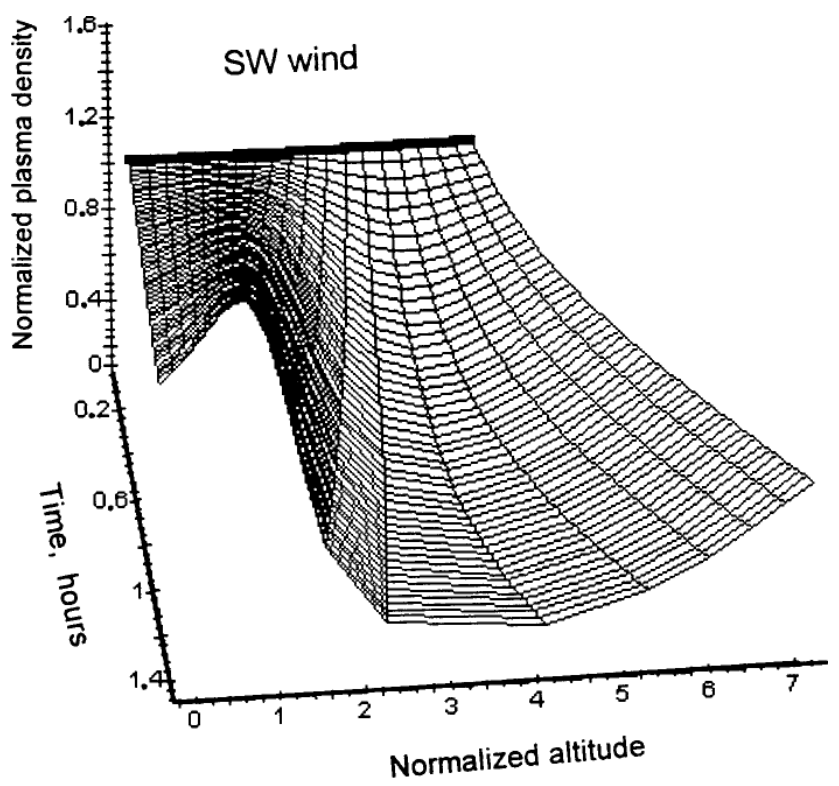

Fig. 7. Same as Fig. 5 but for the northwest PW vortex quadrant, i.e. the profile is representative of $\mathrm{PW}$ winds pointing SW. As seen, a very weak layer forms near $110 \mathrm{~km}$, while the ionization is decreased at higher altitudes.

ion-neutral collisions. Finally, Fig. 8 shows the situation in sector IV, where the wind points in the SE direction. There, both forces act to move ions upward; therefore, the plasma is gradually pushed out of the E-region altitude range under consideration. As seen, a well-defined depletion of ionization develops in less than an hour in the altitudes from $\sim 110$ to $125 \mathrm{~km}$. Obviously, this becomes the most unfavorable sector for sporadic E-layer formation.

We conclude that the effects of vertical plasma transport 


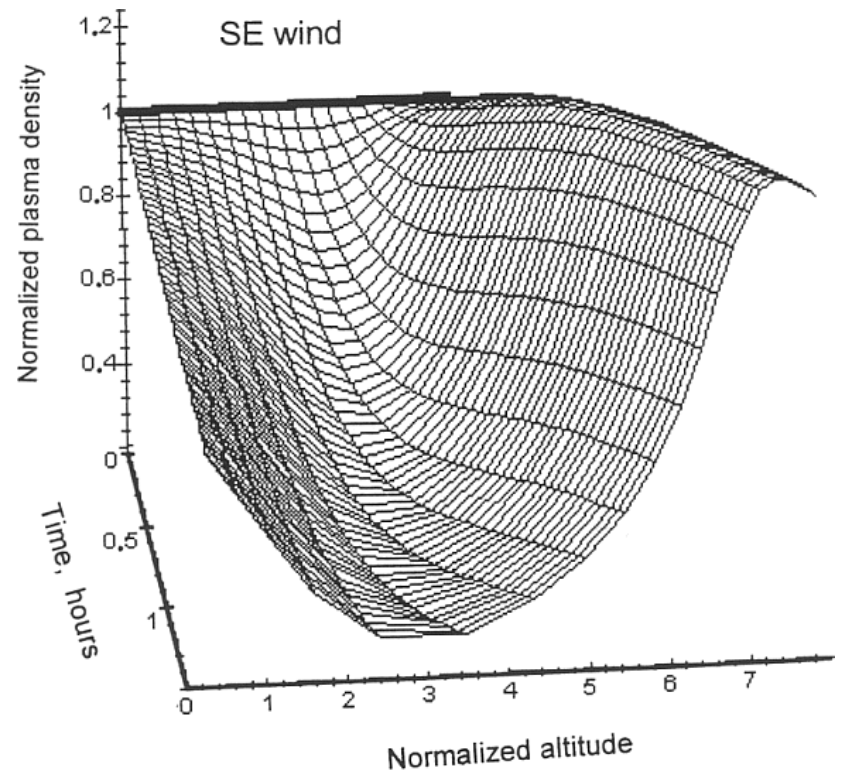

Fig. 8. Same as Fig. 5 but for the southwest PW vortex quadrant, i.e. the profile here is representative of PW wind pointing SE. Here, both vertical forces act to push the ionization out to higher altitudes, thus creating a depletion in the lower E-region.

inside a cyclonic vortex can be quite diverse and complicated. The vertical plasma motions, driven by the large-scale cyclonic wind pattern, tend to redistribute the metallic ion plasma in altitude, which leads to plasma density profiles that have a strong angular dependence. These effects, however, are expected to have relatively short lifetimes compared to the horizontal long-lived plasma accumulation process.

\section{Summary and concluding comments}

This paper provides a starting model for the Shalimov et al. (1999) mechanism of E-region metallic plasma accumulation driven by planetary waves. In the analysis, we considered an elliptical PW vortex of cyclonic wind motion and applied the ion continuity and momentum equations to obtain nonsteady-state solutions and described separately plasma redistribution in both the horizontal and vertical directions.

The non-steady-state numerical solutions in Sect. 3.1 showed that the proposed horizontal plasma accumulation process could increase significantly the metallic ion population inside an area of cyclonic wind motion. This effect, however, depends on altitude; the plasma compression is most effective in the upper E-region, say between 115 and $125 \mathrm{~km}$, where the ion-neutral collision frequency is comparable to the ion gyrofrequency. At lower E-region heights, where $v_{i}$ becomes increasingly higher than $\Omega_{i}$, the ion-neutral frictional forces act to strongly reduce the horizontal Lorentz force action for plasma convergence.

As shown in Sect. 4, vertical plasma transport also exist inside the PW vortex, driven by the combined action of the vertical Lorentz force plus meridional wind ion forcing along the magnetic field lines. Again, the vertical plasma transport was found to be more significant at higher than lower altitudes due to the counteracting role of ion-neutral collisions. The non-steady-state numerical solutions depicted and quantified a horizontal angular dependence for the vertical plasma motions which can set up layer-like density enhancements in one angular sector or valley-like depletions in another. In this picture, the most favorable area for the formation of dense layers at lower E-region altitudes is in the northwest angular quadrant of the $\mathrm{PW}$ vortex, whereas the most unfavorable one is in the southeast quadrant.

It was postulated that the vertical plasma transport effects have much shorter time scales, compared to the horizontal gathering, since they are affected by turbulent mixing inside the E-region. This is likely to occur due to the presence there of additional dynamic neutral wind system components, e.g. internal gravity waves and tidal motions that have smaller temporal and spatial scales but larger amplitudes. These wind components, which were not included in our model, act there independently all the time; thus, it is likely they will constantly modify and, at times, possibly mask entirely the metallic plasma altitude structuring induced by PW vertical plasma transport. Due to turbulent mixing and the much shorter times involved in PW vertical plasma redistribution, its overall effect would tend to average out over periods comparable with PW time scales. On the other hand, since the horizontal plasma convergence persists for long times, it can lead to significant plasma accumulation inside a PW vortex.

By considering both PW-driven, horizontal and vertical plasma motions, the overall picture inside a large area of horizontal plasma convergence, i.e. inside a PW cyclonic vortex, becomes complicated. For short time scales up to a few hours, vertical plasma transport can alter the plasma distribution in part of the area by acting either constructively or destructively to the long-going horizontal PW gathering process. As mentioned above, descending plasma can form dense layers at lower altitudes inside certain PW vortex sectors, whereas in other sectors this effect might be less severe or even become negative, leading to ion depletions. For example, the estimates for a quasi 2-day PW show that after about half a day, the plasma density at $120 \mathrm{~km}$ can rise up to 3 times over an area centered at the PW vortex, with a radius of $\sim 2 \cdot 10^{3} \mathrm{~km}$; on the other hand, in the northwest vortex quadrant, this compression can, in a short time, be enhanced further, up to about 5 times near $110 \mathrm{~km}$, due to vertical transport. Of course, the opposite effect of plasma depletion could prevail in the southeast quadrant, where the horizontally compressed ionization is also subject to flowing out to higher altitudes.

In conclusion, the model presented in this paper shows that the metallic ion accumulation process inside a planetary wave vortex of cyclonic winds is significant, but its effect can be complicated by short-lived vertical plasma transport. Despite these complications, however, there are still large angular sectors inside this huge planetary wave vortex, where horizontal and vertical plasma motions can combine to produce considerable enhancements of metallic ion concentra- 
tion. Naturally, strong sporadic E-layers are likely to occur more easily inside PW sectors of positive vorticity, especially at times when E-region horizontal plasma convergence is reinforced by vertical plasma compression also driven by $\mathrm{PW}$ winds. In this way, although the relationship is more complex and less straightforward than the one suggested first by Shalimov et al. (1999), one still expects to observe a modulation of strong $E_{s}$ occurrence with PW periods, in line with existing observations. However, in order for the horizontal PW accumulation process to be more realistically quantified, the background plasma density, which is taken in this analysis to be constant, needs to be continuously re-adjusted since it keeps changing due to vertical plasma transport alterations and turbulent mixing. We will deal with this through numerical simulations in a future study.

Acknowledgements. This work was supported by the European Office of Aerospace Research and Development (EOARD), Air Force Office of Scientific Research, Air Force Research Laboratory, under contract No. F61775-01-WE004.

Topical Editor M. Lester thanks two referees for their help in evaluating this paper.

\section{References}

Apostolov, E. M., Altadill, D., and Alberta, L.: Characteristics of quasi 2-day oscillations in the foF2 at northern middle latitudes, J. Geophys. Res., 100, 12 163, 1995.

Axford, W. I.: The formation and vertical movement of dense ionized layers in the ionosphere due to vertical wind shears, J. Geophys. Res. 68, 769, 1963.

Forbes, J. M.: Tidal and planetary waves, in: The upper mesosphere and lower thermosphere, a review of experiment and theory, (Eds) Johnson, R. M. and Killen, T. L., p. 67, 1994.

Fraser, G. J.: The 5-day wave and ionospheric absorption, J. Atmos. Sci., 39, 12, 1977.

Gershman, B. N.: Ionospheric Plasma Dynamics, Nauka Publ., Moscow, 1974.

Gossard, E. E. and Hooke, W. H.: Waves in the Atmosphere, Elsevier, Amsterdam, 1975.
Haldoupis, C. and Pancheva, D.: Planetary waves and midlatitude sporadic E-layers. Strong experimental evidence for a close relationship, J. Geophys. Res., 106 (in press), 2002.

Holton, J. R.: An introduction to Dynamic Meteorology, Academic Press., San Diego. Calif., 1982.

Jacoci, C., Schminder, R., and Kürschner, D.: Planetary wave activity obtained from long-period (2-18 days) variations of mesopause region winds over Central Europe $\left(52^{\circ} \mathrm{N}, 15^{\circ} \mathrm{E}\right), \mathrm{J}$. Atmos. Sol. Terr. Phys., 60, 81, 1998.

Kelley, M. C.: The Earth's Ionosphere: Plasma Physics and Electrodynamics, Academic Press., San Diego., Calif., 1989.

Lastovicka, J., Fiser, V., and Pancheva, D.: Long-term trends in planetary wave activity (2-15 days) at $80-100 \mathrm{~km}$ inferred from radio wave absorption, J. Atmos. Terr. Phys., 56, 893, 1994.

MacLeod, M. A.: Sporadic $E$ theory, 1, Collision-geomagnetic equilibrium, J. Atmos. Sci., 23, 96, 1966.

Mathews, J. D.: Sporadic E: current views and recent progress, J. Atm. Solar Terr. Phys., 60, 413, 1998.

Pancheva, D., Alberta, L. F., and De la Morena, B. A.: Simultaneous observations of the quasi-two-day variations in the lower and upper ionosphere over Europe, J. Atmos. Terr. Phys., 56, 43, 1994.

Shalimov, S., Haldoupis, C., Voiculescu, M., and Schlegel, K.: Midlatitude E-region plasma accumulation driven by planetary wave horizontal wind shears, J. Geophys. Res., 104, 28 207, 1999.

Tsunoda R., Yamamoto, M., Igarashi, K., Hocke, K., and Fukao, S.: Quasiperiodic radar echoes from midlatitude sporadic $E$ and role of the 5-day planetary wave, Geophys. Res. Lett., 25, 951, 1998.

Voiculescu M., Haldoupis, C., and Schlegel, K.: Evidence for planetary wave effects on midlatitude backscatter and sporadic Elayer occurrence, Geophys. Res. Lett., 26, 1105, 1999.

Voiculescu M., Haldoupis, C., Pancheva, D., Ignat, M., Schlegel, K., and Shalimov, S.: More evidence for a planetary wave link with midlatitude E-region coherent backscatter and sporadic Elayers, Ann. Geophysicae, 18, 9, 1182-1196, 2000.

Whitehead, J. D.: The formation of the sporadic E-layer in the temperate zones, J. Atm. Terr. Phys. 20, 49, 1961.

Whitehead, J. D.: Recent work on mid-latitude and equatorial sporadic- E, J. Atm. Terr. Phys., 51, 401, 1989.

Zhou, Q. H.: Two-day oscillation of electron concentration in the lower ionosphere, J. Atm. Solar-Terr. Phys., 60, 1669, 1998. 